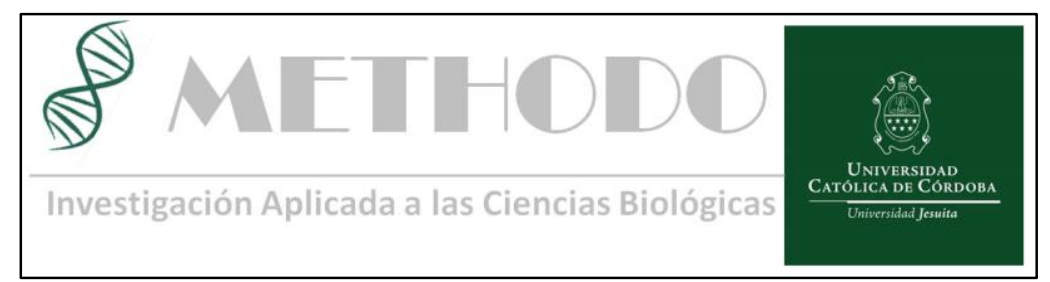

\title{
Hipertiroidismo secundario a adenoma hipofisario productor de TSH. reporte de un caso
}

\section{Secondary hyperthyroidism due to TSH-secreting adenoma. case report}

\author{
Analía V. Checa ${ }^{1}$, Romina A. Gecchelin ${ }^{1}$, María C. Pereyra ${ }^{1}$, Constanza F. Ramacciotti ${ }^{1}$, María L. \\ Bertolino $^{1}$, Mauro J. Pautasso ${ }^{1}$, Fabián C. Saenz ${ }^{2}$, Eduardo N. Cohen ${ }^{1}$. \\ 1 Servicio de Endocrinología. Clínica Universitaria Reina Fabiola, Universidad Católica de Córdoba \\ 2 Servicio de Medicina Interna, Clínica Universitaria Reina Fabiola, Universidad Católica de Córdoba. \\ Correspondencia: Analía V. Checa Servicio de Endocrinología. Clínica Universitaria Reina Fabiola Oncativo 1248-X5004FHP-Cordoba, Argentina; email: \\ avcheca@hotmail.com.
}

\section{Introducción}

El tirotropinoma es un tumor pituitario productor de TSH, $1 \%$ de los adenomas hipofisarios. Es una causa rara de hipertiroidismo. Se presenta con síntomas de tirotoxicosis o masa pituitaria. El 30\% cosecretan otras hormonas ${ }^{1}$. En el laboratorio se evidencia TSH no suprimida con T4 y T3 libres elevadas. Debe realizarse una prueba de TRH seguida de una IRM de hipófisis ${ }^{2}$. El tratamiento de primera línea es quirúrgico ${ }^{3}$.

\section{Caso clínico}

Paciente masculino de 31 años consulta por alopecia universal reciente. Refiere palpitaciones, ansiedad, insomnio e intolerancia al calor. Se constata temblor fino, taquicardia y bocio 1 . Laboratorio: TSH 4,09 (0,27-4,20uUI/ml), T4L $2,51 \quad(0,93-1,71 \mathrm{ng} / \mathrm{dl}), \quad$ T3 $240 \quad(84-202 \mathrm{ng} / \mathrm{dl})$, anticuerpos antiperoxidasa y antitiroglobulina negativos. Andrógenos normales. Ecografía de tiroides: glándula aumentada de tamaño. Test de TRH: TSH a los 30 minutos 4,24uUI/ml (escasa respuesta), sugiriendo hipertiroidismo central. IRM de hipófisis con gadolinio: Imagen hipointensa en $\mathrm{T} 1 \mathrm{~m} \times 12 \mathrm{~mm} 14,6 \mathrm{~mm} \times 6 \mathrm{~mm}$ compatible con adenoma hipofisario. (Figura 1)
Resto de perfil hipofisario normal. Se indica propanolol 40mg/día. Se realiza cirugía con exéresis de adenoma de hipófisis vía transeptoesfenoidal, con buena evolución postquirúrgica y descenso de hormonas tiroideas periféricas hasta alcanzar un valor de T3 115ng/dl, T4L 1,11ng/dl al mes postquirúrgico. Anatomía patológica: Adenoma hipofisario productor de TSH (tirotropinoma). (Figura 2) El paciente presenta recrecimiento de cabello, evoluciona asintomático.

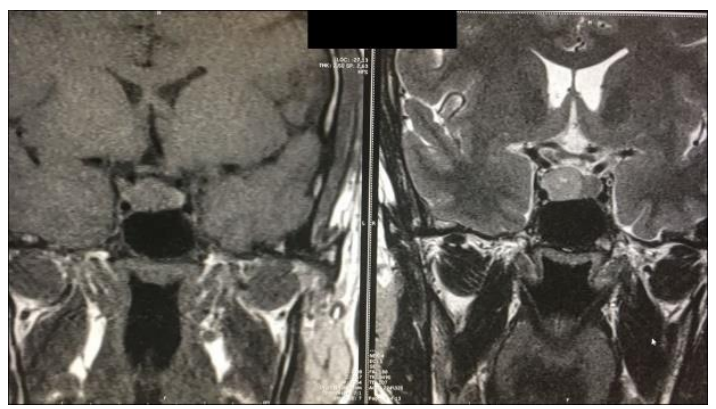

Figura 1: IRM de hipófisis con gadolinio. 


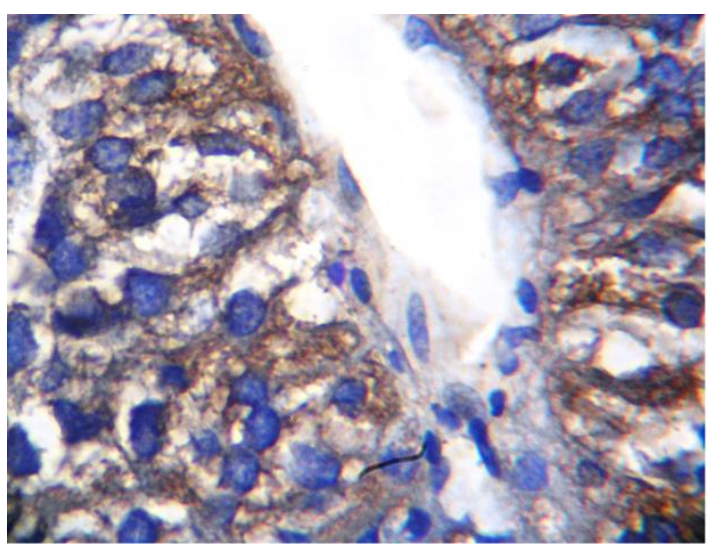

Figura 2: Inmunohistoquímica positiva para TSH en citoplasma.

\section{Conclusión}

El tirotropinoma es un tumor hipofisario de baja frecuencia. La prueba de TRH es de utilidad en el diagnóstico diferencial de tirotropinoma $\mathrm{y}$ resistencia a hormonas tiroideas ${ }^{4}$. Presentamos este caso debido a la baja prevalencia de este tipo de neoplasias y su buena respuesta al tratamiento indicado.

\section{Introduction}

Thyrotropinoma is a TSH-secreting tumor, $1 \%$ of pituitary adenomas. It is a rare cause of hyperthyroidism. It presents with thyrotoxicosis or pituitary mass symptoms. $30 \%$ cosecrete other hormones ${ }^{1}$. Thyroid function tests reveal a not suppressed TSH with high free T4 and T3. TRH stimulation test is the gold standard, completed with a pituitary $\mathrm{MRI}^{2}$. The first line treatment is surgical $^{3}$.

\section{Clinical case}

Male of 31 years who consulted for recent universal alopecia. He referred palpitations, anxiety, insomnia and heat intolerance. Physical examination revealed fine tremor, tachycardia and goiter. Thyroid function tests revealed: TSH 4.09 (0.27-4.20uUI/ml), free T4 $2.51(0.93-1.71 \mathrm{ng} / \mathrm{dl})$ and T3 $240 \quad(84-202 \mathrm{ng} / \mathrm{dl})$ with negative antiperoxidase and antithyroglobuline antibodies. Normal androgen axis. Thyroid ultrasound: enlarged gland. TRH stimulation test: TSH after 30 minutes ${ }^{4} .24 \mathrm{uUI} / \mathrm{ml}$ (low response), suggesting secondary hiperthyroidism. Pituitary MRI: Hypointense image on T1 $12 \mathrm{mmx} 14.6 \mathrm{mmx} 6 \mathrm{~mm}$ compatible with pituitary adenoma. Rest of the pituitary profile was normal. Treatment with Propanolol $40 \mathrm{mg} /$ day was indicated and surgery was performed (transeptosphenoidal exeresis of pituitary adenoma), with well postoperative evolution. Peripheral thyroid hormones decreased until reaching a T3 value of $115 \mathrm{ng} / \mathrm{dl}$, and free T4 of $1.11 \mathrm{ng} / \mathrm{dl}$ a month after patient underwent surgery. Pathological anatomy: TSH producing adenoma (thyrotropinoma). The patient referred hair regrowth and evolved asymptomatic.

\section{Conclusion}

Thyrotropinoma is a low frequency pituitary tumor. The TRH stimulation test is useful in the differential diagnosis of thyrotropinoma and resistance to thyroid hormones ${ }^{4}$. We present this case due to the low prevalence of this type of neoplasms and its well response to initial treatment.

\section{Bibliografía}

1. Yazgan D. et al. Thyrotropinoma and multinodular goiter: A diagnostic challenge for hyperthyroidism. J Res Med Sci. 2013 Nov; 18(11): 1008-1010.

2. Casamitjana r. Actualización de pruebas funcionales en endocrinología. Endocrinol Nutr 2003; 50(6):250-6

3. Pignatta A. et al. TSH Secreting Pituitary Adenoma (Thyrotropinoma): Five Case Reports. Rev Argent Endocrinol Metab 2014; 51:141-150.

4. Singh B. et al. A clinician's guide to understanding resistance to thyroid hormone due to receptor mutations in the $\operatorname{TR} \alpha$ and TR $\beta$ isoforms. Singh and Yen Clinical Diabetes and Endocrinology 2017; 3:8

\section{Palabras claves}

\author{
TIROTROPINOMA, HIPERTIROIDISMO \\ CENTRAL
}

\section{Keywords}

THYROTROPINOMA, CENTRAL HYPERTHYROIDISM.

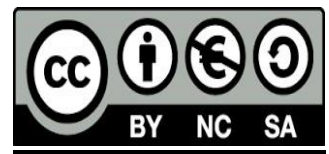

Revista Methodo: Investigación Aplicada a las Ciencias Biológicas. Universidad Católica de Córdoba. Jacinto Ríos 571 Bo Gral. Paz. X5004FXS. Córdoba. Argentina. Tel.: (54) 3514517299 / Correo: methodo@ucc.edu.ar / Web: methodo.ucc.edu.ar | CASO CLINICO Methodo 2019 ;4 (1):25-26 